\title{
Angle transducer based on fiber Bragg gratings able for tunnel auscultation
}

\author{
A. Quintela, J.M. Lázaro, M.A. Quintela, J. Mirapeix, V. Muñoz-Berti, J.M. López-Higuera \\ Grupo de Ingenieria Fotónica - University of Cantabria, \\ Avd de los Castros s/n, 39005 Santander (Spain)
}

\begin{abstract}
In this paper an angle transducer based on Fiber Bragg Grating (FBG) is presented. Two gratings are glued to a metallic platen, one in each side. It is insensitive to temperature changes, given that the temperature shifts affect equally to both FBG. When the platen is uniformly bent an uniform strain appears in both sides of the platen. It depends on the bend angle and the platen length and thickness. The transducer has been designed to be used in the auscultation of tunnels during their construction process and during their live time. The transducer design and its characterization are presented.
\end{abstract}

Keywords: Angle transducer, Fiber Bragg grating, SHM, Tunnel auscultation

\section{INTRODUCTION}

Nowadays civil engineering, and in particular the construction of tunnels, is demanding new ways of monitoring. Such an interest is based on the fact that civil structures and their maintenance are extremely expensive. ..The improvement of the security during the construction process is another reason in this regard. The high costs of maintenance are due to the fact that the monitoring methods used in these days detect the structure's degradation when it is too late, i.e. when physical damage has already occurred.

The photonic sensing technology is this area has a very rapid growth and increasing interest nowadays. This is due in part to devices such as Fiber Bragg Gratings (FBG), whose inherent characteristics make them very suitable for sensing the deformation in civil structures as bridges, dams, buildings, etc ${ }^{1,2}$. Normally in these sensor systems the FBG are used for the measurement of the strain, and it is necessary to implement a method for discrimination between strain and temperature ${ }^{3 .}$

In the case of the tunnel auscultation it could be desirable to have an angle transducer with the advantages provided by the optical sensors based on FBG. In this work a possible transducer solution is presented.

\section{THEORY}

This transducer is based on the same idea used by M.G. Xu et al. ${ }^{4,5}$ for bend measurement, two FBG in booth sides of a platen. When the platen is bent a constant and positive strain appears in the convex side and a negative strain, with the same magnitude, in the concave one. Furthermore, a change of temperature will affect equally to both FBGs. If the bending changes the wavelength shift of both FBGs will change in opposite directions. In addition, if the temperature changes, the wavelength shifts will be similar in both FBGs, therefore it will be very easy to discriminate between temperature and bending. In this paper a transducer that converts angle variations bend variations is proposed.

In a platen with an uniform bend, the strain in the top side in function of the angle of curvature is equal to:

$$
\varepsilon=\frac{L^{\prime}-L}{L}=\frac{d \cdot \alpha}{2 L}
$$

Fourth European Workshop on Optical Fibre Sensors, edited by José Luís Santos, Brian Culshaw, José Miguel López-Higuera, William N. MacPherson, Proc. of SPIE Vol. 7653, 765318

(C) 2010 SPIE · CCC code: $0277-786 \mathrm{X} / 10 / \$ 18 \cdot$ doi: $10.1117 / 12.866268$ 
Where $L^{\prime}$ is the length of the platen top side, $d$ is the thickness an $L$ is the length of the platen. For the bottom side the expression for the strain is the same, but with negative sign. So it is necessary to have more strain for the same angle, to increase the resolution, there are two options: increase the platen thickness or decrease the length of the platen. Probably both FBGs will decrease the dynamic range of the angle transducer. So depending on the desired dynamic range and sensitivity it wil be necessary to choose a platen with a given thickness and length.

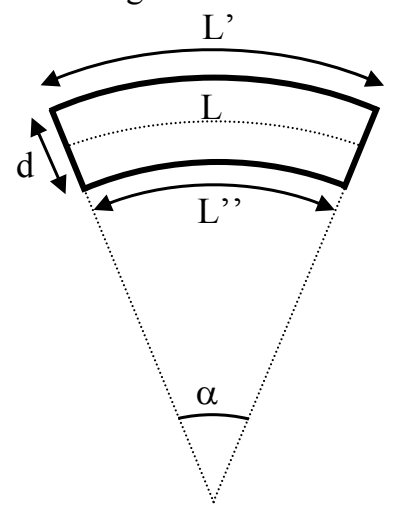

Figure 1. Scheme of the relationship between angle and strain in a bent platen

\section{TRANSDUCER DESIGN}

In Figure 2 a scheme of the proposed transducer is shown. It is composed by two similar pieces that are united by two axes. In the middle of the two pieces there are windows where the metallic platen is fixed with the two glued FBGs, one in each side of the platen. When the relative angle between the two pieces changes, the platen changes its bend, and also the strain in both FBGs.

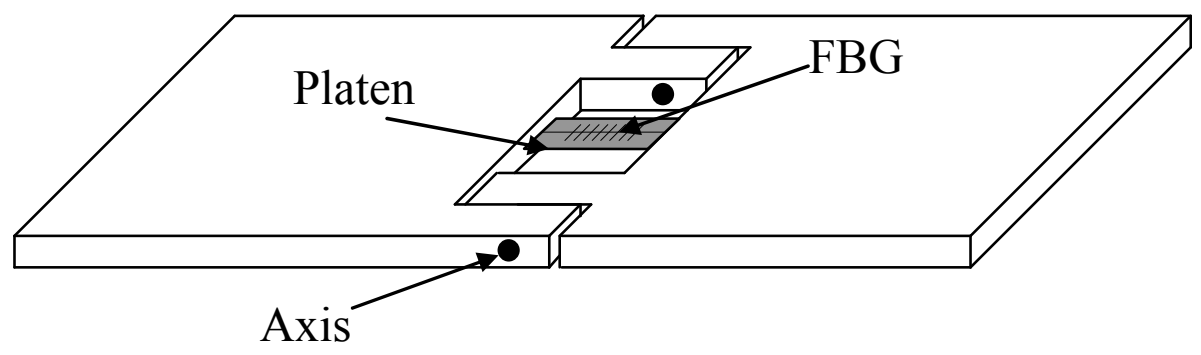

Figure 2. Transducer scheme

This sensor has been designed to be used in tunnel auscultation. One of the possible techniques in this regard is the one presented in Figure 3. It consists of several rigid bars united by the angle transducers, as the one proposed in this work. As the length of the rigid bar and the relative angle between a consecutive pair are known, it is possible to obtain the geometry of a tunnel section, and consequently to check in real time how is their evolution during the fabrication process or during their live time. All angle transducers are connected in series, in the same optical channel. In this case all FBGs must be multiplexed in wavelength. 


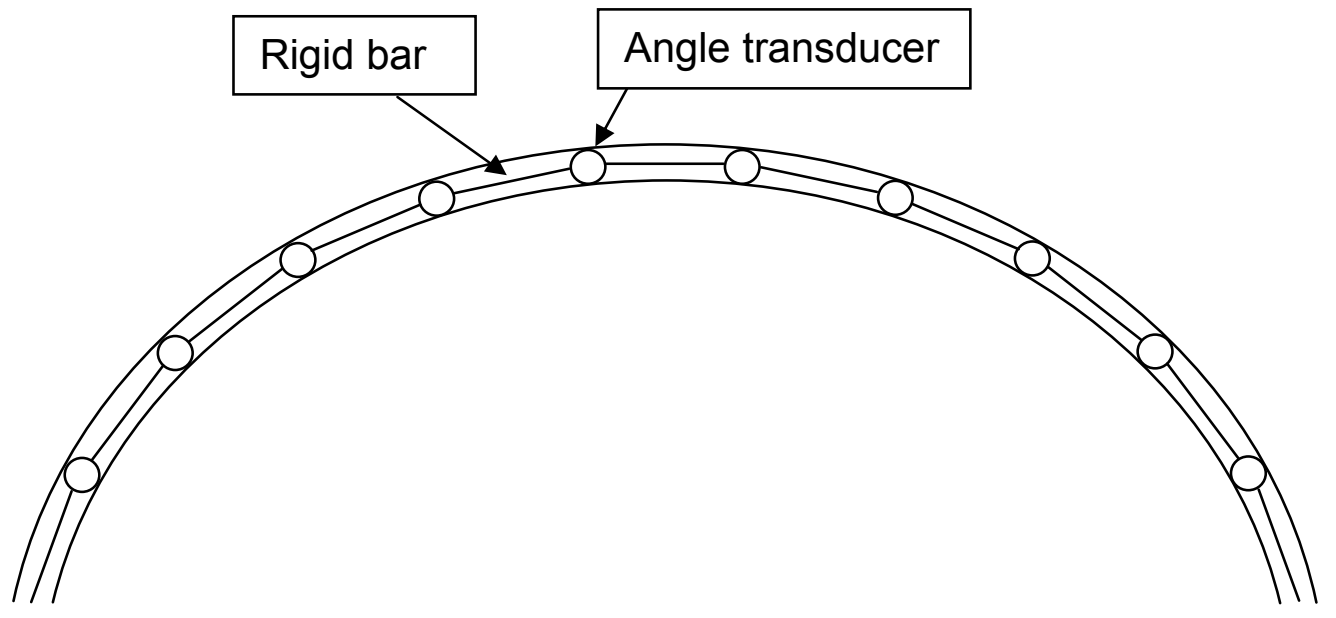

Figure 3. Tunnel auscultation scheme with the proposed transducer.

\section{RESULTS}

The transducer body has been made in aluminum and the metallic platen was of steel. The length of the platen was 10 $\mathrm{mm}$ and the thicknesses 80 micron. Two FBG were glued, one in each side of the platen, using a cyanocrilate glue. The FBG was written using phase masks and a continuous double argon laser $(244 \mathrm{~nm})$ in standard monomode fiber, previously hydrogen loaded.

In Figure 4 two images of the setup used for the measurements are shown. One end of the transducer was fixed to the table and the other to a linear stage that was perpendicular to the transducer. So the linear displacement of the stage produces a change in the angle between the two parts of the transducer body. The wavelength shift has been measured using a FBG interrogator, model BraggMeter from Fibersensing. All the process was controlled by computer.

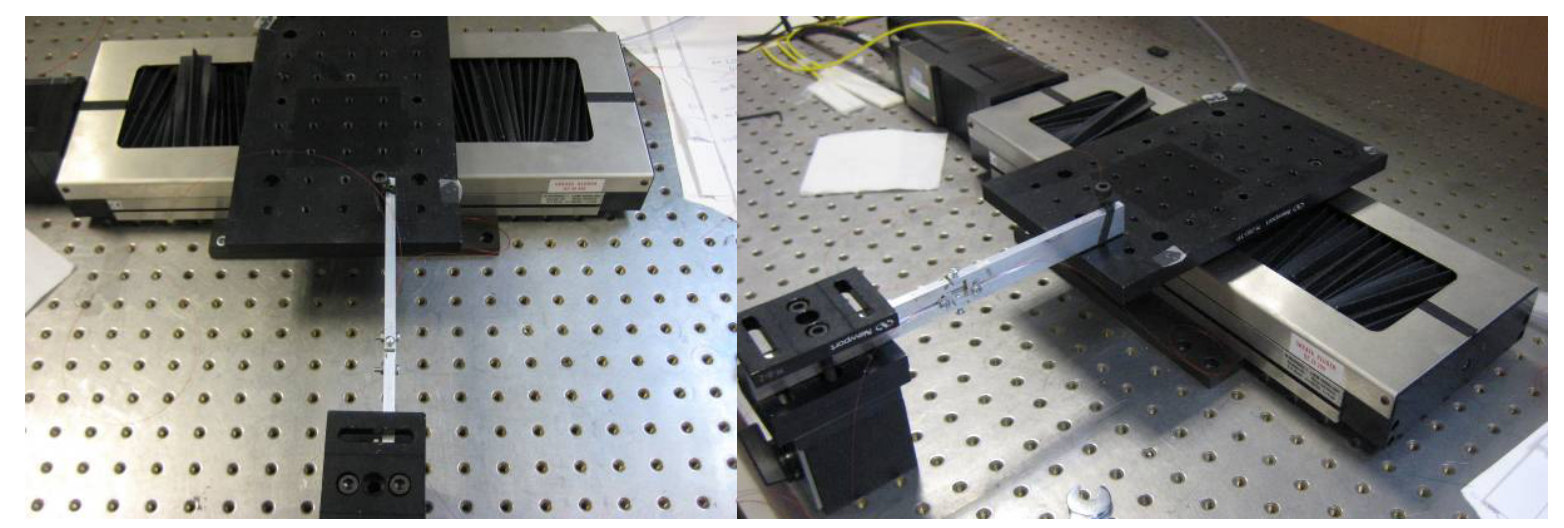

Figure 4. Images of the measurement setup.

The measurement was made from -1.5 to 1.5 degrees with a resolution of 0.01 degree. In Figure 5 obtained results are presented, and the wavelength shifts for both FBGs are also shown. In both cases the response is linear and practically symmetrical. Ideally both FBGs should exhibit the same response but with different sing, although the experimental results are a little different. This is due to the glueing process, given that probably both FBGs were not glued perfectly 
parallel with the platen and neither in symmetrical positions. This is not especially important, because with a previous characterization of the transducers the response of both FBGs is known and it is possible to know from the wavelength shifts the angle of the transducer. In this case, as a FBG interrogation unit with $1 \mathrm{pmm}$ of resolution is used it is possible to obtain a resolution of $3 \cdot 10^{-3}$, what could be enough for the tunnel auscultation.

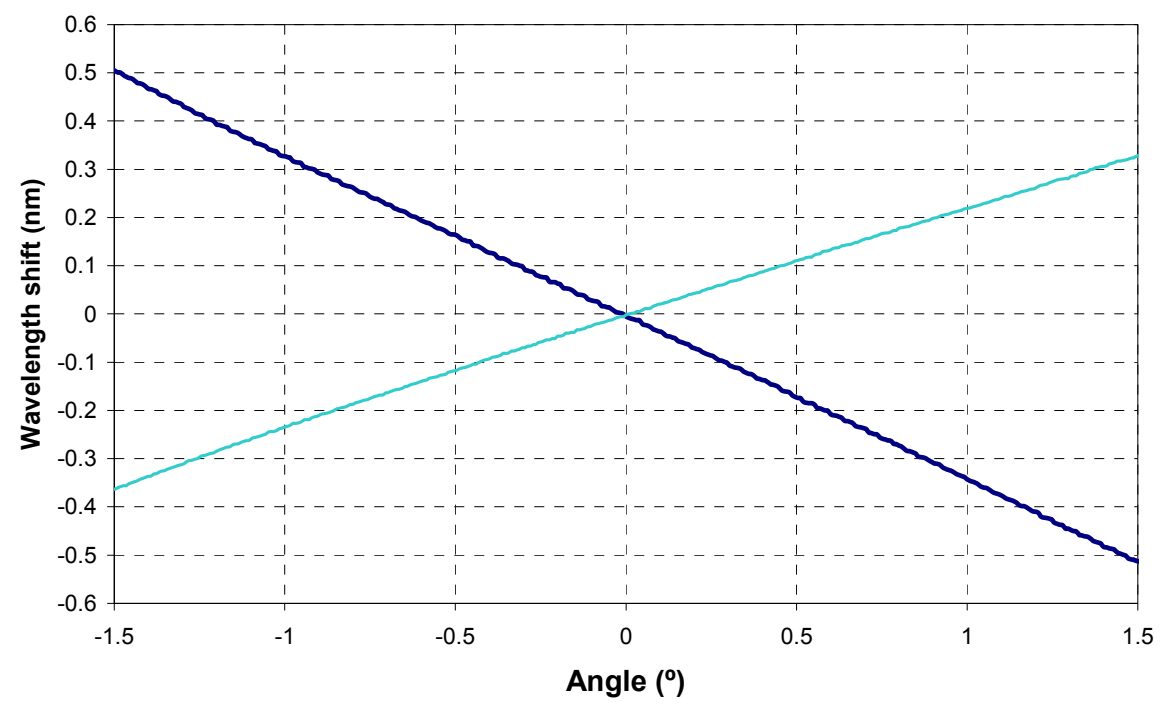

Figure 5. Response to the angle of two transducer's FBG.

\section{CONCLUSIONS}

This work presents an angle transducer based on FBGs able to be used for tunnel auscultation. The transducer consists of a metallic platen, with two FBGs glued, one in each side, and two pieces that transform the variation of angle in changes of the platen bend, and consequently changes in the platen side strains. In this transducer it is very easy to discriminate between strain and temperature, because the response to temperature is equal in both FBGs and the response to the angle it is different. This transducer has been designed, fabricated and characterized. The results obtained show a very linear relation between the angle and the Bragg wavelengths shifts, so it can be use for tunnel auscultation.

\section{ACKNOWLEDGEMENTS}

The authors would like to acknowledge the support provided by the Spanish CICYT through the TEC2007-67987-C02project.

\section{REFERENCES}

1. J. M. López-Higuera, editor. [Handbook of Optical Fiber sensing Technology], Wiley \& Sons, (2002).

2. J. Dakin and B. Culshaw, editors, [Optical fibre sensors: applications, analysis and future trends], Artech House, Boston, (1997)

3. A Quintela , C Jáuregui , J Echevarría and J M López-Higuera, "Embedded temperature-strain fibre Bragg grating sensor system validation for concrete structures," J. Opt. A: Pure Appl. Opt. 4, S387-S390 (2002)

4. M.G. Xu, J.L. Archambault, L. Reekie and J.P. Dakin, "Thermally-compensated bending gauge using surfacemounted fiber gratings.” Int. J. Optoelectron. 9, 281-283(1994)

5. M.J. Gander, W.N. MacPherson, R. McBride, J.D.C. Jones, L. Zhang, I. Bennion, P.M. Blanchard, J.G. Burnett and A.H. Greenaway ,"Bend measurement using Bragg gratings in multicore fibre," Electron Lett. 36 120-121 (2000), 